\title{
L'HOMME L'Home
}

Revue française d'anthropologie

198-199 | 2011

De l'anthropologie visuelle

\section{Corps, décor et envers du décor dans les vidéos populaires africaines}

Jean-Paul Colleyn

\section{(2) OpenEdition}

Journals

Édition électronique

URL : http://journals.openedition.org/lhomme/22672

DOI : 10.4000//homme.22672

ISSN : 1953-8103

Éditeur

Éditions de l'EHESS

Édition imprimée

Date de publication : 25 juillet 2011

Pagination : $33-50$

ISSN : 0439-4216

\section{Référence électronique}

Jean-Paul Colleyn, «Corps, décor et envers du décor dans les vidéos populaires africaines », L'Homme [En ligne], 198-199 | 2011, mis en ligne le 18 juillet 2013, consulté le 30 avril 2019. URL : http:// journals.openedition.org/lhomme/22672 ; DOI : 10.4000/lhomme.22672

Ce document a été généré automatiquement le 30 avril 2019

(c) École des hautes études en sciences sociales 


\title{
Corps, décor et envers du décor dans les vidéos populaires africaines
}

\author{
Jean-Paul Colleyn
}

Bien que, dans l'étude des faits de société, tout acte de naissance soit toujours discutable, de nombreux observateurs s'accordent à dire que Nollywood naquit avec le succès phénoménal, en 1992, de Living in Bondage, une vidéo en ibo sous-titrée en anglais, produite par Kenneth Nnebue et réalisée par Chris Obi Rapu (sous le pseudonyme de Vic Mordi). Elle raconte l'histoire d'Andy, un homme qui quitte la campagne pour la grande ville. Acculé à la misère, il signe un pacte avec le diable pour devenir riche et, dans ce dessein, est amené à sacrifier sa femme. Hanté par son fantôme, il cherche secours dans l'Église. Distribuée en cassettes, cette vidéo atteignit le chiffre de vente record de 500000 exemplaires. Et s'il y a bien des précurseurs au Nigeria, comme Chief Ugbomah et Ola Balogun, le principe de la production vidéo a pourtant sans doute ses racines dans le pays voisin, au Ghana.

2 En 1985, William Akuffo, un distributeur sans aucune expérience de réalisateur, décida d'exploiter la technologie bon marché de la vidéo pour produire des «films». Son premier opus, Zinabu, qui remplit les salles de cinéma et les centres vidéo dans tout le pays, conte l'histoire d'un pauvre mécanicien qui rompt un serment impossible à tenir : en échange de la fortune, il jure de ne pas faire l'amour avec Zinabu, une femme riche et belle, qui l'obsède. Évidemment, il succombe à son désir et le paie de sa vie. À la fin du film, le spectateur apprend que l'ensorcelante Zinabu est véritablement une sorcière.

Le succès de Zinabu, puis de Living in Bondage, a créé un genre, connu sous le sigle «HVF » (Home Video Films), qui n'a cessé de se développer et d'attirer de nouveaux talents. Les apports ghanéens sont incontestables ${ }^{1}$, mais la prolixité et la diffusion de masse de ces vidéos domestiques dépendent fortement de la richesse pétrolière du Nigeria, d'autant plus que les vidéos nigérianes, plus racoleuses (grâce au sexe, à la violence et aux effets spéciaux), n'ont pas tardé à évincer la production « mieux pensante » du Ghana. Dans ces pays, qui ont connu le traumatisme colonial et, pour ce qui est du Nigeria, la guerre civile, les coups d'État militaires, une violence endémique et, depuis 1995, une fragile 
démocratie associée à une incurie de l'État, la vidéo a séduit des millions de spectateurs, n'en déplaise au bon goût des élites.

L'industrie de la vidéo nigériane représente aujourd'hui 290 millions de dollars annuels et quelque 300000 personnes en vivent: 1200 vidéos sont produites chaque année depuis 2005. Cette industrie cinématographique est devenue la troisième du monde, derrière Hollywood et Bollywood, ce qui lui vaut son nom de « Nollywood» (contraction de Nigeria et de Hollywood, expression inventée par un journaliste du New York Times). Mais, à la différence de ces deux derniers foyers de création cinématographique, il ne s'agit pas, au Ghana et au Nigeria, d'une industrie de masse : la plupart des vidéos sont réalisées avec de très petits moyens et de manière artisanale. On ne voit pas encore de grands groupes internationaux comme Vivendi, Universal, Sony ou les majors d'Hollywood s'intéresser aux productions vidéo africaines, mais des connexions internationales se sont établies : implantations malaisiennes et sud-africaines au Ghana et au Nigeria, coproductions Brésil-Nigeria, subsides "African American», sans parler des niches illégales de la mondialisation, comme le piratage à l'étranger ${ }^{2}$. Le coût moyen d'une production tournée en vidéo numérique semi-professionnelle est d'environ 20000\$, y compris les droits de distribution. Elle est réalisée en une dizaine de jours et le « master » est copié en milliers d'exemplaires dans un des innombrables studios créés à cet effet, sur des cassettes VHS ou sur des Video Compact Disc (VCD). Pour un prix de revient de 1,50\$, chaque VCD est revendu le double sur les divers marchés du Nigeria et du Ghana. En termes de réception, le marché ne fait pas défaut, car $70 \%$ des Nigérians ont un magnétoscope ou un lecteur de disques. Sur le plan culturel, l'explosion de cette forme d'art populaire est un phénomène de très grande ampleur qui ne pouvait manquer d'intriguer sociologues, anthropologues, ou spécialistes des médias et du cinéma.

Si la médiocre qualité technique et le caractère stéréotypé des scénarios continuent d'irriter nombre de critiques, incontestablement, un genre audiovisuel nouveau est né, qui a réussi à s'imposer. Certains acteurs et actrices de Nollywood sont devenus de véritables stars médiatisées par les tabloïdes. Sango. The Legendary African King, de Femi Lasode, a été montré au New York African Film Festival en 1998, et The Alexia Affair, de Jeta Amata, a été ovationnée au festival de Berlin 2004, tandis que plusieurs HVF nigérians ont été projetés au $34^{\mathrm{e}}$ Festival du nouveau cinéma de Montréal de 2005. La même année, l'African Movie Academy Awards (AMAA 2005) a créé la surprise en nommant Dickson Iroegbu (The Mayors) «meilleur réalisateur de l'année », son film étant par ailleurs primé pour l'image et le scénario. Toujours en 2005, une conférence se tint à Los Angeles, dans l'idée de réunir des cinéastes nigérians et de potentiels investisseurs ou distributeurs américains (Larkin 2007). En novembre 2008, le directeur du Goethe Institute de Lagos a rendu un vibrant hommage à Nollywood. Deux livres collectifs ont été publiés, avec le concours d'auteurs nigérians: l'un par John Haynes, de l'université de Long Island (Haynes, ed. 2000) ; l'autre par le journaliste et producteur français Pierre Barrot (Barrot, ed. 2005). Il n'a pas manqué d'intellectuels africains pour critiquer ces productions, les traitant de "Juju films" (films de magie et de sorcellerie), propres à perpétuer des stéréotypes préjudiciables pour l'Afrique ${ }^{3}$ (Shehu 1992). On se souvient qu'avant la vogue de Nollywood, le film désormais classique d'ola Balogun, Aiye (1979), qui eut un succès énorme et traite de la sorcellerie, fut critiqué par les intellectuels pour avoir exploité les éléments les plus spectaculaires de la culture yoruba. On pourrait objecter que le fantastique, le surnaturel (y compris dans les films d'horreur, avec, notamment, le retour en force des vampires) et la magie (avec effets spéciaux à l'appui) ont largement envahi 
les écrans occidentaux et dominent tout aussi largement les fictions réalistes, sans qu'on s'inquiète trop de protéger le public. Tous ces genres correspondent bien à notre époque : ils reposent sur le postulat que le monde est mal connu et est peut-être inconnaissable. Tous les intellectuels africains ne rejettent pas le phénomène Nollywood; certains d'entre eux s'y intéressent même de près ${ }^{4}$. Quelques professeurs d'université nigérians et ghanéens sont d'ailleurs passés à l'acte, soit comme réalisateurs, soit comme comédiens.

Le Nigeria compte quelque 120 millions d'habitants répartis dans 56 États et parlant 250 langues. La seule population de Lagos se monte à 13 millions d'habitants. Devant l'impossibilité de couvrir les productions d'une telle fédération, je ne m'intéresserai ici qu'aux vidéos anglophones destinées au public multilingue du Nigeria et du Ghana. L'anglais est en effet aujourd'hui la langue « naturelle » d'un très grand nombre de vidéos et le temps est révolu où elle donnait aux arts du spectacle une allure bourgeoise et artificielle. Le succès des vidéos en anglais, pour la plupart produites par des Ibos, serait notamment dû au fait que cette langue est celle des aspirations sociales ${ }^{5}$. Un grand nombre de films en anglais sont produits par des Ibos, moins soucieux que les producteurs yoruba de s'exprimer dans une langue communautaire, au point que l'on parle souvent des « English Language Igbo Films ».

\section{Public populaire et "quick money"}

5 «Je préfère être un homme d'affaires prospère plutôt qu'un artiste fauché ». C'est par ce slogan bien connu que les producteurs d'Accra me résumaient leur philosophie professionnelle. D'une certaine manière, il s'agit d'une production «de flux», une production de divertissement peu soucieuse de créer du patrimoine. Les films disparaissent en effet très rapidement du marché, car les producteurs escomptent un retour sur investissement rapide, faute de quoi le piratage leur couperait l'herbe sous les pieds. Néanmoins, comme les « séries B » ou les séries télévisées, Nollywood a aujourd'hui ses « classiques $»^{6}$.

6 Au moment où le «bon » cinéma africain (notamment nigérian, soucieux de perfection technique et d'idéaux politiques) ne cesse de décliner - déclin qu'il serait trop long d'expliquer ici -, la vidéo domestique a réussi à créer un espace hautement compétitif qui dépasse la dimension nationale pour s'élever au niveau régional, continental, voire mondial. Alors que de nombreux chefs-d'œuvre du cinéma africain, très dépendants de financements et de distributeurs extérieurs, furent à peine montrés dans les (rares) salles de cinéma des pays concernés, la vidéo s'est développée à partir du public populaire, sans recourir ni au soutien de l'État ni aux techniciens étrangers. La motivation première est essentiellement terre à terre: faire de l'argent rapidement! Quels que soient les jugements portés sur la qualité des scénarios et la richesse de ses messages, cette forme d'expression prouve la capacité de certains pays d'Afrique de produire leurs propres images sans dépendre de l'étranger. Bien que victimes du piratage, il faut reconnaître que de nombreux producteurs et réalisateurs de Nollywood, surtout au début, se souciaient eux-mêmes fort peu du plagiat, du remake ou du piratage des droits d'auteur, tant en ce qui concerne les scénarios que la musique. À l'évidence, il s'agit d'une production à l'écoute du monde, qui s'inspire de tout ce qui se fait et procède d'une « logique métisse ", pour reprendre l'expression de Jean-Loup Amselle (1990).

7 Les nouveaux cinéastes nigérians, très pragmatiques, n'ont aucun problème avec le mercantilisme: ils veulent produire des vidéos qui soient vues par le maximum de 
spectateurs possible. Ayant pris conscience de l'existence, au niveau mondial, d'un grand commerce de l'imagerie, ils veulent s'y faire une place. Le public africain contemporain, quant à lui, apprécie l'aspect ludique et séduisant d'une culture commerciale de l'image largement globalisée (où se croisent mélodrames indiens, films de kung-fu, thrillers américains, telenovelas, etc.), mais il apprécie que l'on produise pour lui des films qui présentent les mêmes attraits tout en puisant dans les traditions locales. Jusqu'à il y a une vingtaine d'années, il ressentait à cet égard une frustration à laquelle les vidéos sont venues répondre.

Comme les tenants du cinéma commercial partout dans le monde, producteurs et réalisateurs déclarent répondre à la demande du public et définissent leur talent par leur capacité à le faire. Ils se soucient moins que leurs aînés d'engagement politique, de plaidoiries en faveur de l'émancipation de l'Afrique, de sauvegarde de son patrimoine ou $\mathrm{du}$ renouvellement du langage cinématographique. C'est pourquoi ce cinéma de divertissement est parfois accusé, à tort, je pense, d'escapism, car, en projetant le spectateur dans l'univers fantasmatique de la fiction, il le distrairait des problèmes du présent et de ses responsabilités citoyennes.

\section{Vidéos et politique}

Que les réalisateurs ne soient pas politiquement engagés ne signifie pas que leurs films soient apolitiques. Nombre d'entre eux sont d'ailleurs produits (intégralement ou partiellement) par des Églises. Par ailleurs, l'écoute attentive d'un public victime de nombreux abus introduit, ne fût-ce que de manière oblique, une dimension politique dans les intrigues de fiction. Les scénaristes s'inspirent très largement des faits divers et des scandales de la politique locale, sans jamais, toutefois, défier ouvertement le pouvoir. La réactivité des vidéastes leur permet de répondre très rapidement aux préoccupations du moment qu'il s'agisse de corruption (Arsenal), d'escadrons para-policiers (Vigilantes, Arsenal, Issabaka), de gangstérisme (Rip Off, Imposters, One Chance, Endtime), de drogue (The last Don, Italian Connection), de crimes rituels (Blood Money, The Vultures Men, Diabolo I-IV, Nkrabea-My Destiny), de trafics d'organes (Blood Money, One Chance), de problèmes de couple (Break Up), de maladies sexuellement transmissibles (Thunderbolt), de sorcellerie ( Women's Cot, Fateful Love, Love in Color, Rising Sun, Fatal Decision), de fantômes (Living in Bondage, He Lives in Me, Ghost Tears, Princess Buttem), ou de sujets historiques comme la guerre civile (Thunderbolt) ou la traite esclavagiste. La question du genre est également omniprésente: les hommes affichent leur pouvoir avec un machisme évident, mais les fortes femmes (qualifiées positivement ou négativement) sont légion. Dans Women's Cot, une association de défense des pauvres veuves est détournée par une sorcière qui la transforme en culte satanique prônant le sacrifice des maris. Heureusement, les militantes modérées reprennent le contrôle de la situation. Sans prendre la forme de réquisitoires, les vidéos décrivent les infidélités systématiques des hommes riches et les adultères en général (Love in Color, The Broken Wall, Chameleon), les violences conjugales (Street Life), les mariages arrangés (Desperate Ambition, Demon-in-Law, Bold Hearts 1\&2), la polygamie (All my Life, Fatal Decision), le chantage sexuel (Chameleon), la prostitution (High Street Girls, My Life Story, Runs, Glamour Girls, Domitilla), le sort peu enviable des veuves ou des femmes sans enfants (Women's Cot, All my Life, The Widow) et les traditions rétrogrades (Evil Forest, Wasted Love, Rituals). De nombreuses vidéos exposent un problème de société récurrent : les troubles dans le système de parenté et les querelles d'héritage (Keep My Will 
, Rituals, Kids are Angry). Dans Emotional Blackmail, un film qui glisse de l'adultère à la fraternité, un inceste du $2^{\mathrm{e}}$ type - deux hommes qui ignorent leur lien de fraternité couchent avec l'épouse de l'un d'entre eux - n'interdit nullement un happy end qui réconcilie tout le monde. Être trahi par "quelqu'un de son sang " apparait comme un ressort dramatique aux effets garantis (Dangerous Twin, Emotional Blackmail, My Life Story, World of Riches). Les troubles de la personne reviennent régulièrement aussi : toujours ambiguë, la personnalité est souvent tiraillée entre l'humain et le non-humain (une instance sorcière, un animal de la brousse, un revenant, un double, etc.). Le conflit de génération permet de cumuler plusieurs de ces thèmes: les mariages arrangés par exemple (Only for Love), les tensions entre marâtre et beaux-enfants (Chameleon). Très souvent, un père autoritaire fait des mauvais choix pour sa fille et finit par lui demander pardon (parfois à genoux) pour ses fautes (Arsenal).

La plupart des intrigues s'inspirent des thèmes de la vie urbaine et/ou villageoise. La « religion » y est presque un cadre de référence obligé. Une majorité de films campe un conflit entre les pouvoirs de la lumière et ceux de l'obscurité. Contrairement à ce que pensaient les théoriciens de la modernité, la religion n'a pas été refoulée dans la sphère privée, sauf peut-être en Europe. Un peu partout les Églises chrétiennes et les confréries musulmanes se sont approprié les médias et interviennent vigoureusement dans la sphère publique. Dans ce duel entre le Dieu chrétien et le Diable, dans les HVF, c'est toujours le premier qui gagne et les agents du mal, en la personne du sorcier d'origine villageoise, qui perdent. Les films jouent généralement sur deux tableaux : ils partagent un message évangélique condamnant les rites occultes, mais, en même temps, se nourrissent des croyances en des sociétés secrètes vouées aux cultes maléfiques (McCall 2002: 86-88; Haynes, ed.: 2000: 4). Comme le remarquait Marc Augé à propos des "prophètes " parfois exorcistes de Côte d'Ivoire, la mise en scène de la lutte contre les démons, sincère ou non, en renforce la croyance plus qu'elle ne la détruit (Augé \& Colleyn 1990). Sur le plan de la scopophilie, le message évangélique se repaît allègrement de ce qu'il dénonce.

\section{"Le paradigme perdu"7}

$9 \quad$ Les grands clivages politiques et culturels du passé restent perceptibles dans toutes les productions culturelles, mais les vidéos anglophones proposent un kaléidoscope de valeurs et de symboles extrêmement vivant. Le plus souvent, la «tradition » n'y est plus invoquée que comme argument conservateur, souvent démenti par les dynamiques économiques, sociales et culturelles. Toutefois, les scénarios exposent la reconfiguration de modèles anciens, malgré l'effet dissolvant du capitalisme et les ruptures proclamées par les Églises nouvelles. Si l'individualisme possessif a instauré la règle «Tout pour atteindre mes objectifs! », les réseaux de relations humaines liés à la parenté, l'alliance, l'ethnie, la religion, restent convoqués, en même temps, par exemple, que la croyance en un destin prénatal ou en une malédiction ancestrale.

10 Contrairement au cinéma africain des années 1960-1990, les vidéos nigérianes et ghanéennes n'idéalisent ni les institutions «traditionnelles» ni l'atmosphère harmonieuse et bucolique des villages; pas plus qu'elles ne diabolisent les mégapoles: malgré toutes ses nuisances, la ville, c'est la civilisation, le village, la brousse. Pas de nostalgie, donc, mais plutôt de la crainte envers ce qui reste du « sacré traditionnel ». Ce qui reste, ce n'est pas tant un ordre social résiduel qu'une réserve de maléfices. N'est 
jamais invoquée une quelconque légitimation mythologique d'une harmonie ancienne, si ce n'est par la figure récurrente du sage, généralement sacrifié. Le village est le lieu d'un savoir dangereux, de l'obscurantisme, du paganisme et de la pauvreté. Le danger de voir les forces obscures de la brousse venir menacer la famille nucléaire, chrétienne et citadine plane en permanence. Si le village reste la source des savoirs occultes, le retour au village se traduit le plus souvent par l'échec. Comme au théâtre, les rituels païens sont toujours caricaturaux et factices, même si une majorité de Nigérians peut les croire plausibles. On ne remarque dans la scénarisation et la mise en scène aucun souci d'ethnographie rigoureuse. Les scènes villageoises sont d'ailleurs presque toujours tournées dans les terrains vagues ou les faux villages des banlieues urbaines. Sous la plume de nombreux auteurs africains contemporains, les films qui traitent de la magie, de la sorcellerie ou de la possession doivent être disqualifiés car ils donnent une mauvaise image du Nigeria (voire de l'Afrique). Toutefois, plutôt que de répudier ces phénomènes comme le font ces auteurs, les réalisateurs et scénaristes les affrontent franchement et en exposent les mécanismes avec plus ou moins de subtilité. Le film One Chance. Ugly Side of City Life, qui affirme se fonder sur des faits réels, explique, par exemple, que les succès et l'impunité du gang One Chance, qui a terrorisé la ville de Lagos, dépendaient de "médecines » préparées par un prêtre d'Igunuko. Les ressorts de la croyance populaire en de telles pratiques méritent certainement qu'on s'y attarde; en tout cas, les producteurs se défendent avec un argument massue : cela intéresse les gens.

\section{La richesse sorcière}

$11 \mathrm{Si}$, pour expliquer les «mystères " de la marchandise productrice de plus-value, Karl Marx invoquait volontiers les «régions nuageuses du monde religieux " (Marx 1969 [1867] : 69), les scénaristes de Nollywood donnent fréquemment à voir les gens riches (souvent des "big men») comme des ogres sans scrupules, si âpres au gain qu'ils recourent au crime pour assouvir leur soif de richesse. Les vidéos ouvrent une lucarne sur une économie sacrificielle digne des rêves les plus débridés de Georges Bataille, avec force dépeçages rituels, dépenses de sang et d'argent. 

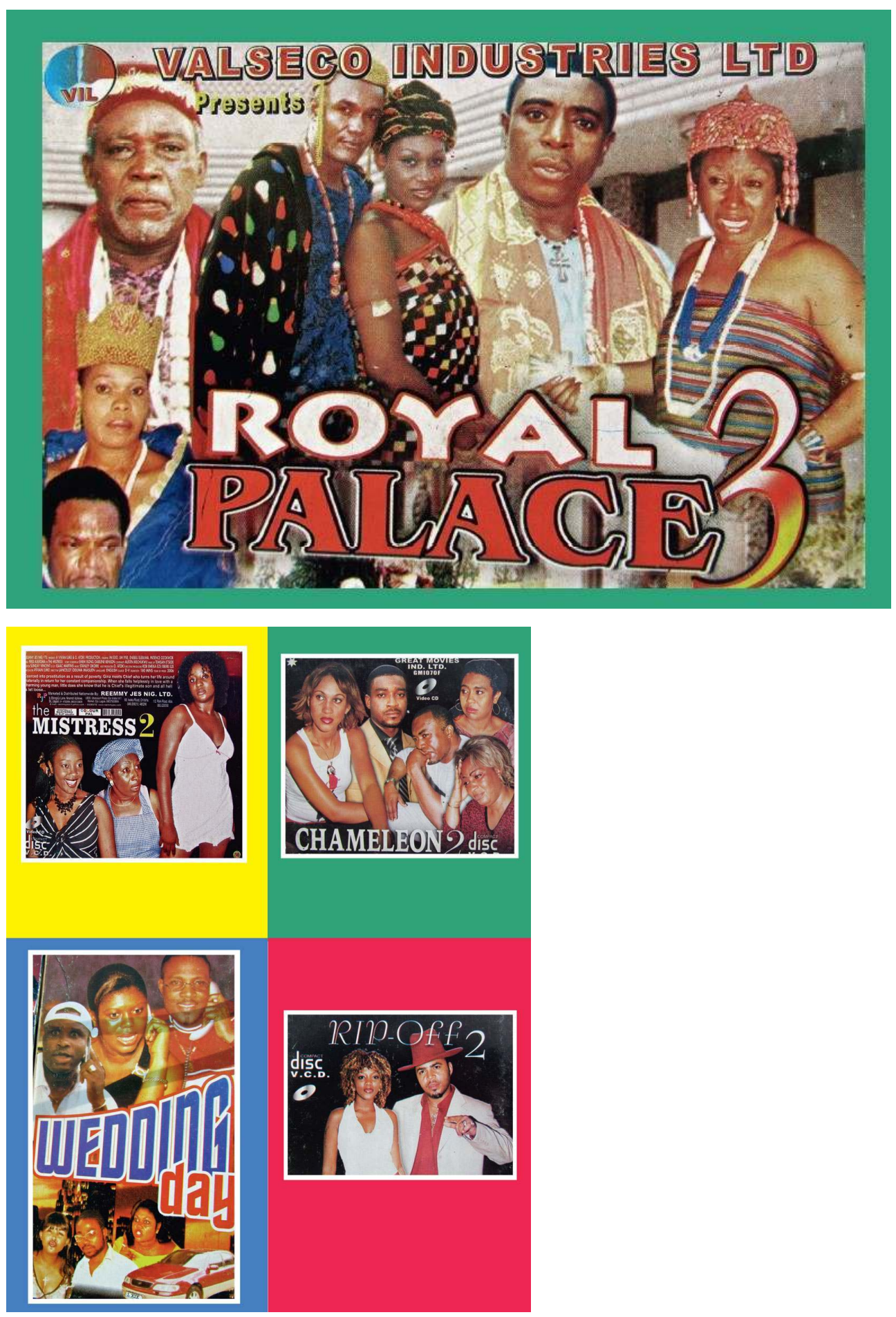

12 Blood Money (Chico Ejiro, Nigeria, 1997) raconte l'itinéraire d'un respectable banquier qui, incapable de rembourser une forte somme qu'il a « empruntée » à sa banque, va glisser progressivement dans le crime. Un officier de police le recommande à Chief Collins, un notable très prospère. Protégé par une police corrompue, Chief Collins se livre en fait au trafic d'organes humains. Il introduit le banquier dans la société des Vautours. Dans un immeuble cossu de Lagos, les Vautours sacrifient rituellement des victimes humaines qui 
vomissent de l'argent. Au héros ainsi devenu tueur en série, on lui demandera bientôt de tuer sa propre mère.

Rituals (Kenneth Nnebue, Nigeria, 1997) s'inspire d'un fait divers connu sous le nom d' Otokoto, lié à la découverte d'un rituel générateur d'argent à Owerri, capitale de l'État d'Imo, en 1996, et qui impliquait le frère d'un ministre. Un « culte » dirigé par un certain Don Pedro, un intellectuel diplômé en sciences politiques à Oxford, exige un sacrifice humain. Après le sacrifice, Don Pedro se livre à une distribution somptuaire d'argent. Un jeune homme décide de s'initier, mais il est contraint d'offrir son propre fils en bas âge en sacrifice ${ }^{8}$.

Francis, le héros de Time (Ifeanyi Onyeabor, Ghana, 2000), s'établit en ville où il plonge dans des difficultés financières inextricables. Désespéré, il retourne au village où un féticheur (jujuman) lui prédit que sa femme va tomber gravement malade, mais qu'il aura de plus en plus d'argent à mesure qu'elle s'affaiblira. Sa femme meurt, mais il cache le corps, car il se met à cracher de l'argent. Il tuera aussi son fils qui a découvert le pot aux roses. À la fin du film, une jeune vierge sur le point d'être sacrifiée sur un autel de brousse invoque Jésus et l'autel est détruit par la foudre.

La série à succès Diabolo I-IV (William Akuffo, Ghana, 1991) met en scène un homme qui se transforme en serpent et pénètre dans le corps des prostituées pour leur faire vomir de l'argent.

16 Dans Nkrabea-My Destiny (Ghana, 1992), qui se passe en milieu rural, un chef sacrifie périodiquement des êtres humains pour perpétuer sa fortune et son pouvoir (en ville). Dans une chambre secrète, un fétiche transforme le sang en argent.

17 Le thème du pacte avec le (ou un) diable en échange de la fortune est un thème récurrent, alimenté par les rumeurs dans les villes du Nigeria et du Ghana. Les sacrifices prescrits exigent très souvent certains organes humains ou un fœetus, ou encore le sacrifice de jeunes enfants. On peut y voir le recyclage du thème ancien de la sorcellerie cannibale, mais c'est aussi l'expression d'une conscience de classe, avec la conviction largement partagée que les pauvres sont aujourd'hui livrés sans défense à des possédants sans scrupule. La dénonciation de la corruption du pouvoir et de la fortune y est claire. L'argent est un problème pour tout le monde : les pauvres parce qu'ils n'en ont pas; les riches parce que leur parentèle et amis convoitent le leur. La richesse masculine - et parfois féminine - s'explique par l'alliance avec un(e) partenaire surnaturel(le), notamment Mamy Wata, la sirène ${ }^{9}$ (Women in Love). De plus en plus, le pouvoir et l'argent apparaissent comme le résultat d'une violence, d'une folie complice de forces occultes. On observe un jeu de correspondances entre magie noire et marché noir, occultisme et économie clandestine, crimes sexuels rituels et production de richesses. De nombreux films - mais pas tous - se concluent par une leçon morale : la foi et la croyance au SaintEsprit apportent le bien-être et même la richesse, plus sûrement que les voies maléfiques (qui ne mènent qu'à la déchéance). Tous les coups ne sont pourtant pas permis, même au Nigeria : chefs locaux, hommes politiques, magistrats ou policiers abusifs peuvent très bien finir en prison. À la fin du film apparait la figure rédemptrice du pasteur, au besoin exorciste, victorieux des forces des ténèbres.

La question de la croyance en ces mythologies modernes est posée (avec toutes ces nuances, du référent historique aux mondes possibles en passant par les rumeurs), mais, quoi qu'il en soit, ces films expriment symboliquement une dénonciation vigoureuse des classes possédantes, considérées comme cannibales ou vampiriques. À noter que les 
sociétés secrètes décrites ressemblent plus à des organisations mafieuses qu'à des cultes traditionnels.

\section{Les films "Alléluia !"} qui pullulent dans la partie sud du Nigeria et qui ont également investi la télédiffusion. Birgit Meyer a souligné les affinités idéologiques entre les messages véhiculés par les HVF ghanéens et les sermons des Églises pentecôtistes, créant ce qu'elle a appelé une « culture publique pentecôtiste» (Meyer 2003). Une bonne partie des vidéos montrées au Ghana provenant, rappelons-le, du Nigeria, la thèse de Birgit Meyer est tout aussi convaincante en ce qui concerne ce pays. Il s'agit toutefois d'une hégémonie diffuse, sans autorité centrale, chaque Église pentecôtiste étant dirigée par une figure prophétique. Utilisant tous les moyens de communication possibles, allant des chaînes de télévision aux HVF, et diffusant à peu près le même message, les Églises exercent une énorme influence dans le cadre de ce qu'on peut appeler un « marché du religieux ».

On ne peut cependant pas affirmer que l'ensemble des films sont lourdement moralisateurs : on y voit évoluer d'authentiques «salauds "; la corruption et les passages à tabac par les policiers sont dépeints comme des pratiques courantes (même si les victimes disposent de quelques recours). Celle qui consiste à user de son influence pour gagner un litige paraît largement l'emporter sur le critère officiel de la légalité. Voici un exemple de la répartition par genre proposée par un grand distributeur: "Action », «Christian », «Comedy », « Drama », « Family », « Ghana », « Horror », « Igbo », « Romance », « Suspense », « Yoruba ».

\section{Esthétique}

Les réalisateurs de Nollywood ne prétendent pas révolutionner l'esthétique cinématographique : ils font du mieux qu'ils peuvent, empruntant librement aux séries et aux cinémas américains, indiens, brésiliens, chinois ou égyptiens ainsi qu'aux différents programmes d'effets spéciaux numériques. Aux productions indiennes, ils adoptent fréquemment le procédé qui consiste en l'utilisation de chansons édifiantes à l'appui de l'image ou du récit, ou comme ponctuation à la manière d'un refrain : "I fell in love long time ago... », " Fruit juice intoxicate when it is served by young ladies ", " Sous les yeux du seigneur ", "Don't break your home, don't break your wife, show her affection », « When we share our dreams... », « You say you're not really satisfied. I need someone like you ", " There is nothing I can do ", " Things are moving faster on the west side ", " Shake your body like a belly dancer!", "Someone is crying, the widow, the widow, the widow... », « Kill him, kill him ! If you kill your husband... ». 


\section{Le décor}

21 La scène sociale des vidéos montre des inégalités flagrantes. Les ascensions sont rapides et spectaculaires, et le luxe des riches outrecuidant. Les films prennent surtout place au sein de la classe urbaine possédante, dans des décors où les pauvres, totalement instrumentalisés, ne font que des incursions. Leur seule échappatoire consiste à s'allier à un puissant ou à menacer de recourir à la sorcellerie. Le spectateur n'appartient pas forcément à l'univers qu'on lui présente et, la plupart du temps, il ne jouit pas des richesses dont les films font étalage. Les décors sont ceux de gigantesques villasforteresses, avec salons luxueux, sofas multiples, tentures de théâtre, home-cinéma (allumé en permanence), champagne, etc. Les déplacements des personnages sont ponctués de sempiternelles ouvertures de grille par un gardien obséquieux convoqué à coups de klaxon. Mais qu'on ne s'y trompe pas : la tragédie guette les riches. Si la misère ne fait évidemment pas le bonheur, l'argent non plus. Dans tous ces films règne une sorte de malédiction sur une fortune ostentatoire et trop vite acquise. Les chutes et les déchéances sont tout aussi rapides que les ascensions sont fulgurantes. Le spectateur peut avoir l'impression de voir la ville (Lagos, Abuja, Accra), mais, en réalité, il n'en voit pas grand-chose. Le réalisateur filme des corps en mouvement, et quelques «travellings voiture ", mais le décor se réduit à fort peu de choses en termes de structures urbaines ${ }^{10}$.

Comme le cinéma en général, celui de Nollywood est un fait social et culturel urbain. Ce dont les producteurs ont besoin, c'est d'être dans un lieu suffisamment dense pour bénéficier d'un réseau de ressources de manière à assurer le dynamisme de la production: finances, gestion, imprimerie, studios, réseau de distributeurs, publicité, presse, etc. Ils ont besoin de ce que Saskia Sassen appelle « urban manufactering » (1996). La ville permet la coordination de toutes ces fonctions.

Accra, Lagos, Abuja, Londres et New York sont en connexion permanente, pour les études des enfants, les affaires, les trafics en tous genres. Elles sont interconnectées bien plus que ne le sont les villes et « leurs campagnes ». Dès qu'une production sort à Lagos, elle se trouve aussitôt à Kano, à Accra, à Kumasi, etc. De même, la classe supérieure de Lagos ou d'Accra est mieux reliée à certains milieux de Londres qu'aux banlieues pauvres de leur ville. Comme unités pertinentes, les villes deviennent plus stratégiques que les nations, les États s'étant débarrassés de la plupart de leurs fonctions sociales, économiques et culturelles. La nouvelle bourgeoisie africaine est internationale : on appelle ceux qui ont voyagé les « Been to » («I have been to London »; « She has been to New York », etc.). Les mondes que l'on voit dans les vidéos sont à la fois très nigérians, ou très ghanéens, et globalisés. Les rôles sociaux les plus fréquemment représentés sont ceux de l'homme et de la femme d'affaires, du médecin ou, d'une manière plus générale, du diplômé (le médecin), du notable local (le «chief»), du pasteur (guide moral, prophète ou faux prophète), de la prostituée (parfois sorcière, parfois au grand cœur), du gigolo.

\section{Le corps}

La mise en scène des corps valorise les embonpoints masculins et féminins comme signes extérieurs de richesse, tout comme les vêtements, les coiffures, les voitures. Les valeurs rendues manifestes par le réalisateur et les acteurs touchent le public sans devoir faire l'objet d'une expression verbale. Dans les images mouvantes se dit quelque chose de plus 
qu'un récit : la manière dont bougent les corps, les différences d'attitudes (entre hommes et femmes, garçons et filles, jeunes et vieux), le statut des enfants (l'intimité physique parents-enfants), les faits de stratification sociale (les signes de soumission, les mimiques du pouvoir), l'iconographie des salons, le rapport à l'argent, à la société de consommation, au voyage. Il serait intéressant de comparer les " techniques du corps » des films du sud du Ghana et du Nigeria avec ceux du Nord, codés par l'islam et la sharia. Certaines scènes deviennent même de véritables motifs, c'est-à-dire des unités repérables, qui se répètent dans le texte filmique et que l'analyste peut isoler. Il pourra ainsi distinguer des motifs iconographiques (la voiture, le home-cinéma, la commande à distance, la jeune fille qui se lime les ongles) et des motifs sociologiques (la femme qui sert à manger à son mari, la fille qui sert son père, l'équation statut masculin ou paternel - richesse). Les HVF montrent de nombreuses scènes de séduction et, ce, pratiquement à tout âge: on voit ainsi des couples d'âge mûr se rouler dans le sable comme de jeunes amoureux, deux amants dîner au bord d'une piscine, des couples endormis (en pyjama) tendrement enlacés, de jeunes mères de famille se promener en pantalon moulant à pattes d'éléphant, une secrétaire gratter le dos de son patron mordu par un insecte, un homme inviter une jeune fille à dîner ( « Je cuisinerai pour toi ! »), une jeune femme offrir une belle montre à l'homme qu'elle veut séduire, une femme inviter un de ses copains à dîner au Sheraton. Voici quelques exemples de séquences: Amanda se rend chez Bryan le soir et lui déclare qu'elle adorerait préparer le petit-déjeuner. En voiture avec Bryan (qui conduit), Amanda lui donne de la crème glacée à la petite cuillère. La radio chante : « No matter what they do. I cannot stop loving you! ». Elle pose la tête sur son épaule et lui nettoie l'oreille // Mark est dans son bain, une superbe baignoire en coin, avec jacuzzi. Sa maitresse (il voudrait l'épouser mais ignore qu'elle est mariée), assise sur le bord, lui caresse les pieds, sous la mousse. Sa main remonte. Il sourit d'aise dans sa mousse. Elle s'agenouille, lui met de la crème douche sur les épaules, le cou. Il l'embrasse, puis l'attire dans le bain. On les voit plus tard dans un bar au son d'une chanson: «Pense aux jours que nous avons vécus. J'ai passé mes jours à penser à nos nuits ». Ils flirtent, boivent du vin rouge (une bouteille pour deux) // Un père frappe à la porte de la chambre de sa fille et entre (elle boude couchée sur son lit) : « Untel vient te demander en mariage. L'amour viendra après ; habille-toi ! ». Il lui confisque son téléphone portable, elle pleure.

La maladie, l'hôpital, la mort sont également des thèmes très présents, mais le drame médical ne se borne pas à un verdict scientifique: non seulement tout le monde, $\mathrm{y}$ compris les médecins, peut être soupçonné d'avoir «injecté » autre chose que le médicament prescrit, mais les mots, voire les intentions, peuvent tuer.

D'une manière générale, un film a doublement à voir avec le corps: d'abord par le traitement du corps - filmer des êtres humains, c'est toujours filmer des corps -, ensuite par les effets sur le corps du spectateur - un film produit des sensations. Dans ces vidéos, on voit fréquemment des hommes pleurer alors qu'ils sont censés contrôler leurs émotions. Si l'on en croit les réactions des blogueurs sur les nombreux sites internet dédiés à Nollywood, la représentation de la violence est mieux acceptée que celle de la sexualité, bien que l'une comme l'autre suscitent des réactions ambiguës : il semble que les censeurs regardent très attentivement ces scènes, pour ensuite protester. Les excès dans l'expression des sentiments sont rarement désapprouvés, comme s'il était évident qu'ils fassent partie du jeu. Ils introduisent un discours parallèle à celui du récit et fonctionnent comme un véritable système. C'est une des raisons pour lesquelles on ne peut analyser ces films vidéos en termes de mimesis par rapport au monde réel. Dans 
l'univers de Nollywood, nous sommes clairement dans la "feintise ludique " (Hamburger 1986; Schaeffer 1999). Remarquons que si le mélodrame se caractérise par un excès d'expression, cet excès est le mieux partagé par de nombreux genres cinématographiques et télévisuels, partout dans le monde.

\section{Question de méthode}

Comme tout fait de culture, la place de la vidéo domestique doit être étudiée dans un jeu d'interactions à la fois politiques, religieuses, morales et esthétiques. Il est possible que les HVF jouent aujourd'hui un rôle comparable à celui joué par les mélodrames dans la culture européenne au moment de la révolution industrielle. L'historien Peter Brooks et puis d'autres ont analysé le mélodrame à la fois comme symptôme et comme instrument $\mathrm{du}$ changement social. Sous l'impact de la révolution, puis du capitalisme, il correspondrait à un moment de dissolution d'une société organique et hiérarchisée, et à une recomposition sur des bases citoyennes nouvelles. Peter Brooks analyse aussi le mélodrame comme un type de système fictionnel qui aide à donner sens à l'expérience (Brooks 1985). Face à une crise de civilisation majeure causée par la violence coloniale et les bouleversements économiques, politiques, culturels qui s'ensuivirent, les fictions vidéos, largement inspirées du monde contemporain, rempliraient le même rôle que les mélodrames, mais par les moyens du cinéma, plus adaptés à perpétuer les principes de l'oralité.

Certains vidéastes de Nollywood participaient déjà à la production de séries télévisées connues sous le nom de soap operas, d'autres sont venus du théâtre, d'autres encore du monde des affaires ou de l'informatique (Okoye 2007). Les vidéos nigérianes et ghanéennes ne sont peut-être pas, par essence, "démocratiques", mais elles ont incontestablement ouvert un espace de débat, aussi bien dans la sphère privée (le cadre familial), que dans la sphère publique, dans les multiples vidéo clubs, à la télévision et sur internet.

L'héritage d'un marxisme trop déterministe et de l'école de Francfort a longtemps invité à considérer toute culture commerciale (les industries culturelles) comme strictement synonyme d'aliénation ${ }^{11}$. Certes, la culture populaire est en partie l'effet des rapports de domination exercés par les classes dirigeantes, mais elle n'est pas le simple reflet de cette domination : elle est une arène où des idées, des représentations, des valeurs s'affrontent, ne fût-ce que parce que la marchandisation recycle tout, y compris la dérision, l'avantgarde, la critique et la contestation. En matière de culture, les frontières sont depuis longtemps brouillées entre le haut et le bas. Au début des années 1960 déjà, Edgar Morin soulignait l'hétérogénéité et le caractère jouissif de la culture de masse (Morin 1962). Le décryptage des HVF par le public est un exercice actif et réflexif. L'opposition entre un cinéma africain " exigeant » (c'est-à-dire élitiste et surtout destiné au "marché » euroaméricain) et la consommation supposée passive de vidéos populaires est aujourd'hui dépassée. Jonathan Haynes a bien montré que l'on pouvait faire une lecture politique de ces films (Haynes 2006).

En ce qui concerne l'efficacité cognitive - quel rôle joue un film dans la compréhension d'un monde? -, il est important de comprendre qu'un film n'est pas un équivalent dévalué d'un discours verbal: il est outil de pensée (Aumont 1996). Les «images» 
filmiques défient toujours l'analyste, car l'image est une notion polysémique : il peut s'agir de l'image en tant qu'objet d'une représentation mentale ou d'une construction idéologique. Selon la Gestalttheorie, les structures élémentaires de la pensée, l'idéation sont déjà partiellement des images, des mixtes d'iconique et de symbolique. Y a-t-il une pensée sans métaphore? On peut en douter, car la métaphore est une opération fondamentale de l'esprit. Pour pouvoir s'énoncer, la moindre idée recourt à la métaphore. Du coup, le problème de l'image comme reflet d'un référent se pose de manière cruciale, car on peut douter qu'un référent quelconque soit jamais accessible sans la médiation d'une figuration. Toute prétention à la mimesis a donc quelque chose d'un coup de force et la neutralité de l'apparence n'est qu'une illusion. Dans tout univers humain, les objets sont des signes. Les HVF ne sont ni une fenêtre ouvrant directement sur les grandes villes de la côte ouest-africaine ni des élucubrations débridées de l'imagination, mais des productions historiques, situées dans un temps et un lieu liés à une évolution technique, à une conjoncture économique, politique et culturelle. Ils ne reflètent pas fidèlement le monde, mais ils en font le commentaire et influencent, au niveau individuel comme au niveau collectif, le devenir. On sait aujourd'hui que la réception d'un film ou d'un programme télévisé est de nature ambiguë, car il faut tenir compte en effet du plaisir de l'interprétation: un spectateur peut apprécier au niveau ludique un programme dont il désapprouve les contenus ou dont il déplore certains aspects. Il peut réfléchir aux circonstances de sa propre vie sans se projeter naïvement dans les personnages de fiction.

\section{Apprendre sans que nous n'en sachions rien}

Sur le plan esthétique, la colonisation a laissé un héritage pesant en ce sens qu'elle a fait du cinéma une instance très fermée, orientée vers le contrôle et la pédagogie lourde. Elle a, de ce fait, programmé une contre-propagande, elle aussi tout entière vouée à la persuasion. Sur ce plan, les vidéos marquent une rupture franche, bien qu'elles ne soient pas toujours immunes de tout prêche. Indépendamment de l'histoire, du drame raconté, avec son début, son milieu et sa fin, les HVF disent quelque chose de la société, de ceux qui les ont réalisés, de leurs destinataires qui les plébiscitent ou les boudent. L'anthropologue notera, par exemple, la tentation assez massive de proposer un nouvel univers moral, une nouvelle sphère publique sous le contrôle de Dieu, contre l'avidité propre aux tentations d'un monde "moderne » associé aux forces du mal venues de la brousse. Mais ce qui l'intéresse n'est pas au premier chef le récit, même si les «messages » d'un film gardent tout leur intérêt. Si peu réalistes qu'elles soient, les vidéos rendent bien compte de la longue crise rampante que vivent les sociétés africaines contemporaines. Ce nouvel univers moral est constamment sous la tension des désirs personnels (assez freudomarxistes avec les relations entre sexualité transgressive et richesse) et des tentations des « affaires» (un terme lui-même équivoque). Même si elles illustrent la grande force de l'individualisme, ces vidéos ne participent, ni du côté de Dieu ni de celui des maléfices, d'un désenchantement du monde. 


\section{BIBLIOGRAPHIE}

Adejunmobi, Moradewun, 2002 «English and the Audience of an African Popular Culture : The

Case of Nigerian Video Film », Cultural Critique 50 : 74-103.

Amselle, Jean-Loup, 1990 Logiques métisses. Anthropologie de l'identité en Afrique et ailleurs. Paris, Payot («Bibliothèque scientifique »).

Augé, Marc \& Jean-Paul Colleyn, 1990 Nkpiti. La rancune et le prophète. Paris, Éd. de l'École des hautes études en sciences sociales (" Anthropologie visuelle»).

Aumont, Jacques, 1996 À quoi pensent les films ? Paris, Séguier.

Barrot, Pierre, ed., 2005 Nollywood. Le phénomène vidéo au Nigeria. Paris, L’Harmattan («Images plurielles »).

Brooks, Peter, 1985 [1976] The Melodramatic Imagination. Balzac, Henry James, Melodrama and the Mode of Excess. New York, Columbia University Press.

Hamburger, Kate, 1986 Logique des genres littéraires. Paris, Le Seuil. [Éd. orig. allemande : 1957.]

Haynes, Jonathan, 2006 « Political Critique in Nigerian Video Films », African Affairs 105 (421) : 511-533.

Haynes, Jonathan, 2007 « Nnebue : The Anatomy of Power », Film International 5 (4) : 30-43.

Haynes, Jonathan, ed., 2000 Nigerian Video Films. Athens, Ohio University Center for International Studies.

Larkin, Brian, 2007 « The Nollywood Rising Conference », Film International 5 (4) : 109-111.

McCall, John C., 2002 « Madness, Money, and Movies : Watching a Nigerian Popular Video with the Guidance of a Native Doctor », Africa Today 49 (3) : 79-94.

McCall, John C., 2007 «The Pan-Africanism We Have : Nollywood's Invention of Africa », Film International 5 (4) : 92-97.

Marx, Karl, 1969 [1867] Le Capital, I. Paris, Garnier-Flammarion.

Meyer, Birgit, 2003 « Ghanian Popular Cinema and the Magic in and on Film », in Birgit Meyer \& Peter Pels, eds, Magic and Modernity. Interfaces of Revelation and Concealment. Stanford, Stanford University Press.

Morin, Edgar, 1962 L'Esprit du temps. Paris, Grasset.

Okoye, Chukwuma, 2007 « Looking at Ourselves in our Mirror : Agency, Counter-Discourse, and the Nigerian Video Film », Film International 5 (4) : 20-29.

Sassen, Saskia, 1996 Losing Control ? Sovereignty in an Age of Globalization. New York, Columbia University Press.

Schaeffer, Jean-Marie, 1999 Pourquoi la fiction ? Paris, Le Seuil (« Poétique »).

Shehu, Brendan, 1992 No... Not Nollywood. Essays and Speeches of Brendan Shehu. Ed. by Hyginus Ekwuazi \& Yakubu Nasidi. Jos, Nigeria Film Corp. 


\section{NOTES}

1. Birgit Meyer (de l'Université d'Amsterdam) a étudié les prémices ghanéennes du phénomène ; au Nigeria, on peut expliquer la genèse du genre par le théâtre ambulant yoruba et les soap de la télévision.

2. Ce secteur en expansion souffre beaucoup du piratage. La « Motion Picture Association of America » rapporte que plus de 20 millions de VCD et 4,5 millions de cassettes vidéo piratées ont été saisis en 2000, mais les vidéastes n'ont pas les moyens d'engager des poursuites judiciaires. Les films piratés vendus par milliers dans le Bronx, à Brooklyn ou à Washington, ne rapportent pas un centime à ceux qui les réalisent (Washington Post du 20 novembre 2003).

3. Juju : recours aux esprits, à la magie, aux rituels sondés sur des savoirs occultes, soit à des fins de protection, soit à des fins d'agression.

4. Notamment Sola Fosudo (Université d'État de Lagos), Ayo Akinwale (Université de Ilorin), Laz Ekwueme (Université de Lagos), Chukwuma Okoye (Université de Ibadan), Onookome Okome (Université d'Alberta).

5. Pour une analyse nuancée du recours à l'anglais, lire Moradewun Adejunmobi (2002).

6. Distribués par Amazon au prix exorbitant de 25US \$.

7. Titre d'Edgar Morin qui évoque un temps où nature et culture travaillaient de concert : Le Paradigme perdu. La nature humaine, Paris, Le Seuil, 1973.

8. Pour une analyse du film, cf. Haynes (2007).

9. Souvent représentée sous les traits d'une femme blanche, à partir d'une iconographie indienne.

10. Lire à ce sujet : Kerstin Pinther, s.d. «City Without Features on the Production of the Urban in Ghanian and Nigerian Video » (http://www.learningfrom.com/pinther.html).

11. Selon John McCall, l'erreur d'Adorno dans sa critique du jazz devrait faire réfléchir ceux qui rejettent en bloc la vidéo populaire (cf. 2007 : 96).

\section{RÉSUMÉS}

Résumé

Dans les années 1990, le Ghana et le Nigeria ont connu une véritable révolution culturelle sous la forme d'un genre cinématographique nouveau, totalement indépendant des sponsors étrangers. Brassant des centaines de millions de dollars, fabriquant des stars, et captivant un public de masse, des vidéos familiales, connues sous le nom de Nollywood, se sont hissées au $3^{\mathrm{e}}$ rang mondial de l'industrie cinématographique. Décriés par les esthètes pour leur caractère commercial et leur côté «bas de gamme ", ces films traitent pourtant de problèmes de sociétés qui intéressent tout le monde : l'individualisme, la réussite sociale, les aléas du couple, la parenté, l'héritage, le népotisme, la corruption, la sorcellerie, les traditions rétrogrades, les confits 
religieux, l'exode, la maladie, la mort, le destin. Si peu réalistes qu'elles soient, ces vidéos rendent bien compte de la crise que vivent les sociétés africaines contemporaines, tout en infirmant les théories du désenchantement du monde.

Abstract

In the 1990s, Ghana and Nigeria experienced a literal cultural revolution in the form of a new type of cinema completely independent from foreign sponsors. Involving hundreds of millions of dollars, manufacturing stars and captivating a mass audience, family videos known as « Nollywood» rose to the third place in the film industry worldwide. Belittled by aesthetes as too commercial and "downscale», these films dealt with social problems of interest to everyone : individualism, social success, the ups and downs in the life of a couple, kinship, inheritance, nepotism, corruption, witchcraft, backward traditions, religious conflicts, migrations, illness, death, destiny. Regardless of how much they lacked in realism, these videos clearly depicted the crisis of contemporary African societies while invalidating theories about the disenchantment of the world.

\section{INDEX}

Keywords : Nollywood, African Cinema, Video Films, Popular Culture, Nigeria, Ghana

Mots-clés : Nollywood, cinéma africain, films vidéo, culture populaire, Nigeria, Ghana

\section{AUTEUR}

\section{JEAN-PAUL COLLEYN}

École des hautes études en sciences socialesCentre d'études africaines (CEAf), Paris colleyn00@gmail.com 